\author{
Universidade de São Paulo \\ Escola de Enfermagem de Ribeirão Preto
}

Aeromicrobiota do ambiente cirúrgico: princípios e peculiaridades da climatização artificial

Juliana Ferreira Lima de Paula

RIBEIRÃO PRETO

2003 


\author{
Universidade de São Paulo \\ Escola de Enfermagem de Ribeirão Preto
}

\title{
Aeromicrobiota do ambiente cirúrgico: princípios e peculiaridades da climatização artificial
}

\author{
Juliana Ferreira Lima de Paula
}

Dissertação de mestrado, apresentada à Escola de Enfermagem de Ribeirão Preto da Universidade de São Paulo, para obtenção do Título de Mestre em Enfermagem, no Curso de Pós-Graduação em Enfermagem - Área de concentração: Enfermagem Fundamental - Linha de Pesquisa: Doenças Infecciosas: Problemática, especificidade e estratégias de enfrentamento.

Orientadora

Prof $^{a}$ Dra. Denise de Andrade

RIBEIRÃO PRETO 
FICHA CATALOGRÁFICA

Paula, Juliana Ferreira Lima

Aeromicrobiota do ambiente cirúrgico: princípios e peculiaridades da climatização artificial. Ribeirão Preto, 2003.

p.111: il. ; $30 \mathrm{~cm}$

Dissertação de Mestrado, apresentada à Escola de Enfermagem de Ribeirão Preto/USP - Área de concentração: Enfermagem Fundamental.

Orientadora: Andrade, Denise de.

1. Infecção hospitalar. 2. climatização 3. ar condicionado 4 . ortopedia 


\section{Autorização para reprodução}

Autorizo a reprodução e/ou divulgação total ou parcial da presente obra por qualquer meio convencional ou eletrônico, desde que citada a fonte.

Juliana Ferreira Lima de Paula

Universidade de São Paulo

Escola de Enfermagem de Ribeirão Preto

Departamento de Enfermagem Geral e Especializada

Av: Bandeirantes, 3900. Monte Alegre

Ribeirão Preto - SP, 18 de dezembro de 2003.

Email: julianaflima@ig.com.br 
Data da defesa:

1

\section{Banca Examinadora}

Profa. Dra. Denise de Andrade

Julgamento:

Assinatura:

Profa. Dra. Izabel Yoko Ito Julgamento: Assinatura:

Profa. Dra. Maria Cristina Galvão Julgamento: Assinatura: 


\section{DEDICATÓRIA}

À Deus pelo dom e graça da vida, presença constante em meu caminho.

À Antônio (in memoriam), meu pai, exemplo de simplicidade, honestidade e dedicação à família. Infelizmente não pode presenciar o resultado deste trabalho. Saudades...

À Vanda, minha mãe, minha companheira de todos os momentos, minha eterna gratidão.

À Alexandre, meu marido, inestimável companheiro e profissional. Meu alento nos momentos difíceis e que soube compreender minhas ausências, ajudando-me a transpor todas as dificuldades do caminho.

À Marina, minha filha querida, que contribuiu para que entendesse o verdadeiro significado da vida.

Aos meus irmão, Marcelo e Mateus, cúmplices de todos os momentos, por acreditarem em mais uma conquista de minha vida.

"Assim como falham as palavras quando querem exprimir qualquer pensamento; assim falham os pensamentos quando querem exprimir qualquer realidade" (Fernando Pessoa) 


\section{AGRADECIMENTOS ESPECIAIS}

À Profa. Dra. Denise de Andrade, por acreditar em meu projeto, pela paciência e respeito aos meus momentos e limites, pela amizade e apoio. Exemplo de competência, profissionalismo, dedicação e entusiasmo pela pesquisa.

À Profa. Dra. Izabel Yoko Ito, pela inestimável contribuição para a realização deste projeto, pela disponibilidade, paciência e sabedoria. 


\section{AGRADECIMENTOS}

À Profa. Dra. Maria Cristina Galvão, pela contribuição valiosa para a realização deste trabalho.

À Evandro Watanabe, pela colaboração na coleta das amostras no centro cirúrgico do HCFMRP - USP.

Ao Engenheiro Luciano Niciolli, pelo suporte técnico, disponibilidade e atenção.

Ao laboratório de Microbiologia do departamento de Análises Clínicas Toxicológicas e Bromatológicas da Faculdade de Ciências Farmacêuticas de Ribeirão Preto da Universidade de São Paulo, pela realização da etapa laboratorial.

Ao setor de engenharia do HCFMRP - USP, pela concessão do material para pesquisa, bem como pelo suporte técnico.

Aos funcionários do centro cirúrgico do HCFMRP - USP pela colaboração durante a coleta dos dados.

À Deolinda e Lurdes pelo auxílio nas buscas de referências bibliográficas.

À todos aqueles que contribuíram de alguma forma para a realização deste trabalho.

Muito obrigada. 


\section{Resumo}

PAULA, J. F. L. Aeromicrobiota do ambiente cirúrgico: princípios e peculiaridades da climatização artificial. 2003. 111 p. Dissertação de Mestrado - Escola de Enfermagem de Ribeirão Preto da Universidade de São Paulo.

O presente estudo tem como meta primordial investigar a temática aeromicrobiota do ambiente hospitalar, especialmente, do centro cirúrgico com vistas a enfocar contaminação biológica por conseqüência da climatização artificial, bem como fornecer informações técnicas aos profissionais afins. Assim, estabeleceu-se como objetivos específicos: quantificar e categorizar a produção do conhecimento científico nacional e internacional relacionada a aeromicrobiota do ambiente hospitalar e testar um método de avaliação microbiológica do ar do ambiente cirúrgico considerando os parâmetros biológicos da legislação nacional, e o binômio custo/benefício. As autoras evidenciaram na literatura investigada que a avaliação microbiológica do ar é expressiva nos estudos, e, quanto ao tipo de microrganismo o Aspergillus $\mathrm{sp}$ e o Staphylococcus $\mathrm{sp}$ foram os mais mencionados. Em seguida, apontam a problemática da aerocontaminação biológica e discutem as alternativas de controle da qualidade do ar. Com relação a avaliação microbiólogica do ar por meio de placas Petrifilm ${ }^{\mathrm{TM}} \mathrm{AC}-$ Aerobic Count (3M, St Paul, MN, USA) em diferentes salas cirúrgicas ortopédicas observaram que o número de amostras positivas da entrada foi menor que na saída do ar: sala1, 0,0 na entrada e 55,6 na saída; sala 2, 40 e 83,3 e na 14 de 20,0 e 60,0\%, respectivamente. Quanto ao nível de contaminação (ufc) foi expressiva apenas duas amostras da sala 2. No computo geral, os gêneros isolados foram Bacillus, Micrococcus, Actinomyces. Vale acrescentar que fungos não foram detectados. Esses resultados apontam para a eficiência do sistema de climatização. Alertam para a necessidade de uma pluralidade de ações estruturadas na multidisciplinaridade, bem como, na aplicação crítica das Resoluções e Portarias vigentes.

Palavras chaves: Aeromicrobiota, Infecção Hospitalar, Risco biológico, Centro Cirúrgico 


\title{
Air microbiology of surgical environment: principles and singularities of artificial climate.
}

\begin{abstract}
This study has as main goal to investigate about air microbiology of hospital environment, specially, surgical center, aiming to focus biological contamination due to artificial climate, as well as to provide technical information to professionals. So, the specific goals of this study are to quantify and classify national and international production of scientific knowledge related to air microbiology of hospital environment and test an evaluation method of air contamination, taking into account biological parameters of Brazilian legislation, and the relationship between cost and benefit. The authors have pointed out into scientific literature that air microbiological evaluation is expressive and the microorganisms Aspergillus and Staphylococcus were mentioned mostly. Next, they show the problems concerning biological contamination by air and discuss about alternative ways of controlling air quality. Regarding the microbiological air evaluation applying Petrifilm $^{T M}$ AC - Aerobic Count plates (3M, St Paul, MN, USA) in different orthopedic surgical rooms, the results reveal a greater number of positive samples collected at the air exit compared to those at the air entry: room 1 got 0.0 at entry and 55.6 at exit; room 2, 40 and 83.3 and room 14, 20.0 and 60.0\%, respectively. The contamination level (ufc) was expressive only on two samples of room number 2. The final analysis shows that the isolated genus were Bacillus, Micrococcus and Actinomyces and fungus were not detected. These results point out the efficiency of the air conditioning system and strengthen to the need of a plurality of structured actions in the multidisciplinary field, as well as in the appliance of Brazilian legislation.
\end{abstract}

Key words: Air microbiology, hospital infection, biological risk, surgery center. 


\section{Aeromicrobiota del ambiente quirúrgico: principios y peculiaridades de la climatización artificial.}

\section{Resumen}

El presente estudio tiene como meta primordial, investigar la temática aeromicrobiota del ambiente hospitalario, especialmente del centro quirúrgico, con vistas a enfocar la contaminación biológica como consecuencia de la climatización artificial, bien como a proveer informaciones técnicas a los profesionales afines. Así, se establecieron como objetivos específicos: cuantificar y categorizar la producción de conocimiento científico, nacional e internacional relacionada a la aeromicrobiota del ambiente hospitalario y probar un método de evaluación microbiológica del aire y del ambiente quirúrgico, considerando los parámetros biológicos de legislación nacional, y el binomio costo-beneficio. Las autoras encontraron evidencia en la literatura investigada, acerca de los resultados de la evaluación microbiológica del aire, en cuanto a los tipo de microorganismos, como son: el Aspergillus sp y el Staphylococcus sp, que fueron los más mencionados. Enseguida, apuntan a la problemática de la aerocontaminación biológica y discuten las alternativas de control de calidad del aire. Con relación a la evaluación microbiológica del aire por medio de placas Petrifilm ${ }^{\mathrm{TM}} \mathrm{AC}-$ Aerobic Count (3M, St Paul, MN, USA), en diferentes salas quirúrgicas ortopédicas, se obtuvo como resultado, que el número de muestras positivas de la entrada fue menor que en la salida del aire: sala 1, 0.0 en la entrada y 55.6 en la salida; sala 2, 40 y 83.3 y en la 14 , de 20.0 y $60.0 \%$, respectivamente. En cuanto al nivel de contaminación (ufc) se expresó solamente en dos muestras de la sala 2. En el cómputo general, los géneros aislados fueron: Bacillus, Micrococcus y Actinomyces. Es oportuno informar que no se detectaron hongos. Estos resultados apuntan a la eficiencia del sistema de climatización. Alertan para la necesidad de una pluralidad de acciones estructuradas en la multidisciplinariedad, así como a la aplicación crítica de Resoluciones y Normas vigentes.

Palabras claves: Aeromicrobiota, Infección Hospitalaria, Riesgo Biológico, Centro Quirúrgico. 
